\title{
La aplicación del control interno en las organizaciones de sector no financiero de la economía popular y solidaria del Ecuador
}

\author{
The application of internal control in non-financial sector organizations of \\ the popular and solidarity economy of Ecuador
}

Recibido: 10-03-2019 / Revisado: 15-04-209 /Aceptado: 24-05-2019/ Publicado: 05-06-2019

Jorge Luis Chafla Granada. ${ }^{1}$, Viviana Catalina Solís Morejón. ${ }^{2}$, Marcelo Eduardo Sánchez Salazar. ${ }^{3}$, Edison Ruperto Carrillo Parra. ${ }^{4} \&$ Roberto Fabián Sánchez Chávez. ${ }^{5}$

\begin{abstract}
DOI: $\underline{\text { https://doi.org/10.33262/cienciadigital.v3i2.6.592 }}$

The Social and Solidarity Economy in Ecuador has become the main factor in boosting the country's finances, its products and services are the benchmark for the acceptance of people, its coverage and presence have generated change in the productive matrix of the strategic sectors and seek to combine good living with collective participation. It has served to apply the group economy, generating market expansion strategies allowing applying the priority of work over capital and collective interests over individual interests. In such reason the present the objective of this study is to demonstrate the application of the components of internal control in the Non-Financial Sector Organizations of Ecuador, for which the analysis of each of the bodies that make up these organizations is proposed, describing the attributions and responsibilities defined in the Law. Organic of Popular and Solidarity Economy and its Regulation, determining a common definition of internal control in response to the needs of the different governing bodies and executors of these organizations.
\end{abstract}

In this area, the interaction of its theoretical-methodological supports constitutes a guide for social and solidarity economy research, and suggests the theoretical basis of COSO I, II and III for the members of cooperatives and associations of the real sector from an area of knowledge of the economic and social sciences; whose conclusions open future studies on the importance of promoting models

\footnotetext{
${ }^{1}$ Escuela Superior Politécnica de Chimborazo, Facultad de Administración de Empresas, Riobamba, Ecuador, jorge.chafla@espoch.edu.ec

${ }^{2}$ Escuela Superior Politécnica de Chimborazo, Facultad de Administración de Empresas, Morona Santiago, Ecuador, viviana.solis@espoch.edu.ec

${ }^{3}$ Escuela Superior Politécnica de Chimborazo, Facultad de Administración de Empresas, Morona Santiago, Ecuador, marcelo.sanchez@espoch.edu.ec

${ }^{4}$ Escuela Superior Politécnica de Chimborazo, Facultad de Administración de Empresas, Riobamba, Ecuador, edison.carrillo@espoch.edu.ec

${ }^{5}$ Escuela Superior Politécnica de Chimborazo, Facultad de Administración de Empresas, Riobamba, Ecuador, roberto.sanchez@espoch.edu.ec
} 
and matrices for an integral analysis of control of the institution in areas of competitiveness, risk reduction and promoting sustained economic growth since the creation of a social and solidarity organization appears as an alternative to the serious problem of unemployment that the country is going through.

For this reason it is vital to establish an Internal Accounting Control based on policies, procedures that relate to the protection of assets and the reliability of financial records and therefore to obtain reasonable security in all operations that are executed in organizations With this procedure, managers based on financial and administrative information can make timely and reasonable decisions.

Keywords: Internal Control, Popular and Solidarity Economy, Non-Financial Sector

\section{RESUMEN}

La Economía Social y Solidaria en el Ecuador se ha convertido en el factor principal en la dinamización de las finanzas del país, sus productos y servicios son el referente para la aceptación de las personas, su cobertura y presencia han generado cambio en la matriz productiva de los sectores estratégicos y buscan conjugar el buen vivir con la participación colectiva. Ha servido para aplicar la economía de grupo, generando estrategias de expansión de mercado permitiendo aplicar la prelación del trabajo sobre el capital y de los intereses colectivos sobre los individuales. En tal razón el presente estudio tiene como objetivo demostrar la aplicación de los componentes del control interno en las Organizaciones del Sector No Financiero del Ecuador, para lo cual se propone el análisis de cada uno de los órganos que integran estas organizaciones, describiendo las atribuciones y responsabilidades definidas en la Ley Orgánica de Economía Popular y Solidaria y su Reglamento, determinando una definición común del control interno en respuestas a las necesidades de los diferentes órganos de gobierno y ejecutores de estas organizaciones.

En este ámbito la interacción de sus sustentos teóricos-metodológicos constituye una guía para las investigaciones en economía social y solidaria, y sugiere la base teórica del COSO I, II y III para los integrantes de cooperativas y asociaciones del sector real a partir de una área de conocimiento de las ciencias económicas y sociales; cuyas conclusiones dan apertura a futuros estudios sobre la importancia de promover modelos y matrices para un análisis integral de control de la institución en áreas de competitividad, reducción de riesgo y fomentar el crecimiento económico sostenido ya que la creación de una organización social y solidaria aparece como una alternativa al grave problema del desempleo que atraviesan el país.

Por tal motivo es de vital importancia establecer un Control Interno Contable basado en políticas, procedimientos que se relacionen con la protección de los activos y la confiabilidad de los registros financieros y por consiguiente para obtener seguridad razonable en todas las operaciones que se ejecuten en las organizaciones, con este procedimiento los directivos en base a la información financiera y administrativa pueden tomar decisiones oportunas y razonables.

Palabras Claves: Control Interno, Economía Popular y Solidaria, Sector No Financiero 


\section{Introducción}

Toda organización, sea grande o pequeña requiere de control interno con el objetivo de garantizar la idoneidad de sus operaciones. Es conveniente destacar que el Control Interno es un plan coordinado de todos los métodos y procedimientos para:

- Proteger los activos.

- Obtener información correcta y segura.

- Promocionar la eficiencia de operación.

- Regirse a las políticas prescritas por la normativa vigente.

Esta investigación pretende detallar los elementos básicos del control interno que deben desarrollar las organizaciones no financieras de la Economía Popular y Solidaria, para garantizar un sistema de control interno que asegure la gestión eficiente y económica de la organización.

El Manual de control interno establece los procesos a seguir por las organizaciones. Un elemento fundamental de este proceso lo constituye el autocontrol que es responsabilidad del Consejo de Vigilancia y del auditor interno y externo.

\section{Metodología}

Metodológicamente, a partir del método teórico, se fundamenta los hechos y fenómenos de variable que intervienen en el control interno, permitiendo crear premisas para comprender la correlación con el control aplicado en las organizaciones de la economía popular y solidaria.

Por tanto, es necesario entender los fundamentos teóricos del análisis de las funciones del Control Interno en las organizaciones no financieras (ONF) de la Economía Popular y Solidarias del Ecuador. En el sentido amplio de la palabra es la ciencia de las leyes que rigen la producción y el intercambio en la sociedad humana la ciencia de las condiciones y las formas bajo las que producen y cambian lo producido las diversas sociedades humanas". (Engels 1963:179)

La función cognoscitiva de la Economía Popular y Solidaria, se orienta hacia el estudio del control interno en las organizaciones no financieras; representa el sistema de conocimientos que sustenta la teoría de dicha ciencia.

En esta misma línea, la función metodológica, por su parte, está dirigida a servir de fundamento teórico del resto de las ciencias económicas, tanto de aquellas que explican los aspectos funcionales y sectoriales de la sociedad, como de ciencias económicas que se relacionan con otras ramas del conocimiento.

Razón por la cual, la función cognoscitiva como la metodológica no pueden ser analizadas de manera indistinta a la función práctica ya que esta refleja las acciones prácticas de la sociedad en el proceso de transformaciones económicas, sociales y políticas.

En base a lo expuesto, el proceso metodológico utilizado en el control interno de las ONF, fundamenta su interpretación a partir de la función ideológica de la EPS.

Desarrollo. 


\section{Control interno}

Según Alfonso Armador Sotomayor, (2008), con referencia al Control, menciona lo siguiente: "El concepto de control, como una etapa del proceso administrativa, es decir, funcional, representada una actividad de vigilancia del cumplimiento de las operaciones y la confirmación del apego a la normativa establecida, así como los objetivos planeados". (Pág. 25)

Según, Pacheco, (2008), al control lo define como: Un mecanismo que permite corregir desviaciones a través de indicadores cualitativos y cuantitativos dentro de un contexto social amplio, a fin de lograr el cumplimiento de los objetivos claves para el éxito organizacional, es decir, el control se entiende no como un proceso netamente técnico de seguimiento, sino también como un proceso informal donde se evalúa factores culturales, organizativos, humanos y grupales. (pág. 4)

Según (Casares, 2013) Con el informe COSO (COMMITTEE OF SPONSORING ORGANIZATIONS), de 1992, se modificaron los principales conceptos del Control Interno dándole a este una mayor amplitud.

El Control Interno se define entonces como un proceso integrado a los procesos, y no un conjunto de pesados mecanismos burocráticos añadidos a los mismos, efectuado por el consejo de la administración, la dirección y el resto del personal de una entidad, diseñado con el objeto de proporcionar una garantía razonable para el logro de objetivos.

La seguridad a la que aspira solo es la razonable, en tanto siempre existirá el limitante del costo en que se incurre por el control, que debe estar en concordancia con el beneficio que aporta; y, además, siempre se corre el riesgo de que las personas se asocien para cometer fraudes.

Se modifican, también, las categorías de los objetivos a los que está orientado este proceso.

De una orientación meramente contable, el Control Interno pretende ahora garantizar:

- Efectividad y eficiencia de las operaciones.

- Confiabilidad de la información financiera.

- Cumplimiento de las leyes y normas que sean aplicables.

- Salvaguardia de los recursos.

\section{Importancia}

El control interno cumple un papel relevante y substancial en la conducción, organización, mediante el control e información de sus actividades, contribuyendo con lo siguiente:

- Utilización eficiente y protección de los recursos humanos, materiales, financieros y tecnológicos disponibles en la empresa.

- Producir información contable, financiera y administrativa confiable, real y oportuna, fijando y evaluando los procedimientos respectivos, que ayudan a la empresa en la toma de decisiones, para lograr sus objetivos. 
- Detectar y controlar los riesgos de errores e irregularidades existentes en la empresa, identificar sus causas y proponer acciones correctivas factibles, evaluando todos los niveles de autoridad, la administración del personal, los métodos y sistemas contables.

- Cumplimiento de las políticas, leyes, reglamentos y procedimientos aplicables para la realización de sus operaciones, para que éstas sean desempeñadas de forma correcta y oportuna, contribuyendo a alcanzar los objetivos de la organización. (Mantilla, 2016)

\section{Características}

- El sistema de control interno forma parte integrante de los sistemas contables, financieros, de planificación, de información y operacionales de la respectiva entidad.

- Corresponde a la máxima autoridad del organismo o entidad, la responsabilidad de establecer, mantener y perfeccionar el sistema de control interno, el cual debe ser adecuado a la naturaleza, estructura y misión de la organización

- En cada área de la organización, el funcionario encargado de dirigirla es responsable por el control interno ante su jefe inmediato de acuerdo con los niveles de autoridad establecidos en cada entidad.

- La unidad de control interno es la encargada de evaluar en forma independiente el sistema de control interno de la entidad y proponer el representante legal del respectivo organismo las recomendaciones para mejorarlo. (Galvis, Montes Salazar, \& Mejía Soto, 2007)

\section{Objetivo}

El objetivo del control interno, es proporcionar a la empresa un grado de seguridad razonable en cuanto al logro de objetivos.

El control interno ha sido diseñado para ayudar a organizar, controlar, evaluar y mejorar las políticas, los métodos y procedimientos de la empresa, además de asegurar la obtención de información financiera confiable, oportuna y suficiente como herramienta útil para la gestión y el control.

Los objetivos del control interno se ubican dentro de las siguientes categorías:

- Operaciones: Este se relaciona de manera directa con la misión y visión de la empresa y a la vez determina el uso efectivo y eficiente de los recursos financieros, materiales y humanos con los que cuenta la empresa.

- Información financiera: Se refiere a la elaboración de Estados Financieros que sean claros, confiables y oportunos de igual manera útiles para la toma de decisiones.

- Cumplimiento: se relaciona con el cumplimiento por parte del personal de la empresa a las determinadas leyes, políticas y regulaciones a las que se encuentra sujeta.

De acuerdo a estas categorías los objetivos del control interno más importantes son: 
- Proteger los activos de la empresa contra pérdida, fraudes o ineficiencia

- Crear políticas que permitan el respeto, cuidado, protección y administración, de los bienes de la institución.

- Promover la exactitud y confiablidad de los informes contables y administrativos.

- Proveer políticas que impulsen la correcta operación de la entidad

- Evaluar la mejor utilización del recurso humano y tecnológico

- Contar con políticas sólidas y bien estructuradas que permita una administración y operación ordenada.

- Asegurar el cumplimiento de las regulaciones legales y normas reglamentarias de las disposiciones administrativas.

- Realizar un organigrama que determine las líneas de autoridad y dependencia con sus respectivos niveles jerárquicos establecidos en la entidad. (Mantilla, 2016)

\section{Objetivos del control interno}

Para Madariaga, (2004), los objetivos de Control Interno, son:

1. Salvaguardar los bienes de la empresa evitando pérdidas por fraude o por negligencia.

2. Comprobar la exactitud y veracidad de los datos contables, los cuales son usados por la dirección para tomar decisiones.

3. Promover la eficiencia de la explotación.

4. Estimular el seguimiento de las prácticas ordenadas por la gerencia. (pág. 67)

Para Cardozo (2008), los objetivos del control interno son:

- Prevenir fraudes.

- Descubrir hurtos o malversaciones.

- Obtener información administrativa, contable y financiera, confiable, segura y oportuna.

- Localizar errores administrativos, contables y financieros.

- Proteger y salvaguardar los bienes, valores, propiedades y demás activos de la entidad.

- Promover la eficiencia del personal.

- Detectar desperdicios innecesarios tanto de material, tiempo y recursos en general.

- Mediante su evaluación, graduar la extensión del análisis, comprobación y estimaciones de las cuentas y operaciones sujetas a auditoría por parte del auditor.

- Detectar los riesgos inherentes a la cartera de crédito, riesgo administrativo, financiero, como de la plataforma tecnológica. (págs. 33-34)

\section{Componentes del control interno}

Para (Mantilla, 2007), los componentes de control interno son:

\section{Ambiente de control.}

La esencia de cualquier negocio es su gente - sus atributos individuales, incluyendo la integridad, los valores éticos, y la competencia - y el ambiente en el que opera. La gente es el motor que dirige la entidad y el fundamento sobre el cual todas las cosas descansan. 


\section{Valoración de riesgos.}

La entidad debe ser consciente de los riesgos y enfrentarlos. Debe señalar objetivos, integrados con ventas, producción, mercadeo, finanzas y otras actividades de manera que opere concertadamente. También debe establecer mecanismos para identificar, analizar y administrar los riesgos relacionados.

\section{Actividades de control.}

Se deben establecer y ejecutar políticas y procedimientos para ayudar a asegurar que se están aplicando efectivamente las acciones identificadas por la administración como necesarias para manejar los riesgos en la consecución de los objetivos de la entidad.

\section{Información y comunicación.}

Los sistemas de información y comunicación se interrelacionan. Ayudan al personal de la entidad a capturar e intercambiar la información necesaria para conducir, administrar y controlar sus operaciones.

\section{Monitoreo.}

Debe monitorearse el proceso total, y considerarse como necesario hacer modificaciones. De esta manera el sistema puede reaccionar dinámicamente cambiando a medida que las condiciones lo justifiquen. (pág. 18)

\section{Métodos de evaluación del control interno}

Según Cardozo, (2008),manifiesta que existen tres métodos de evaluación:

\section{Método Descriptivo}

Consiste en la explicación por escrito de las rutinas establecidas para la ejecución de las distintas operaciones o aspectos científicos del control interno. Es decir, es la formulación mediante un memorando donde se documenta los distintos pasos de un aspecto operativo.

\section{Método de Cuestionario}

Consiste en elaborar previamente una relación de preguntas sobre los aspectos básicos de la operación a investigar y a continuación se procede a obtener las respuestas a tales preguntas. Generalmente estas preguntas se formulan de tal forma que una respuesta negativa advierta debilidades en el control interno.

\section{Método Gráfico}

Este método tiene como base la esquematización de las operaciones mediante el empleo de dibujos; en dichos dibujos se representan departamentos, formas, archivos y en general el flujo de la información de las diferentes operaciones hasta afectar la cuenta del mayor general.

En la práctica éste resulta ser el método más eficiente para estudiar y evaluar el control interno, ya que se parte de la nada y toda la información es producto de la observación, investigación, inspección e indagaciones directas que efectúa el auditor. (págs. 38-39) 


\section{Componentes}

Figura 1: Cubo COSO 1992 vs COSO 2013

coso 1992

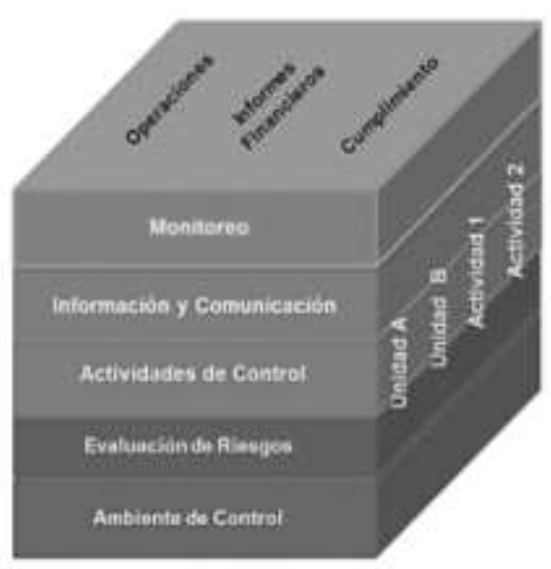

Fuente: (Buzo, 2014, pág. 3)

Elaborado por: Los Autores

\section{$\operatorname{coso} 2013$}

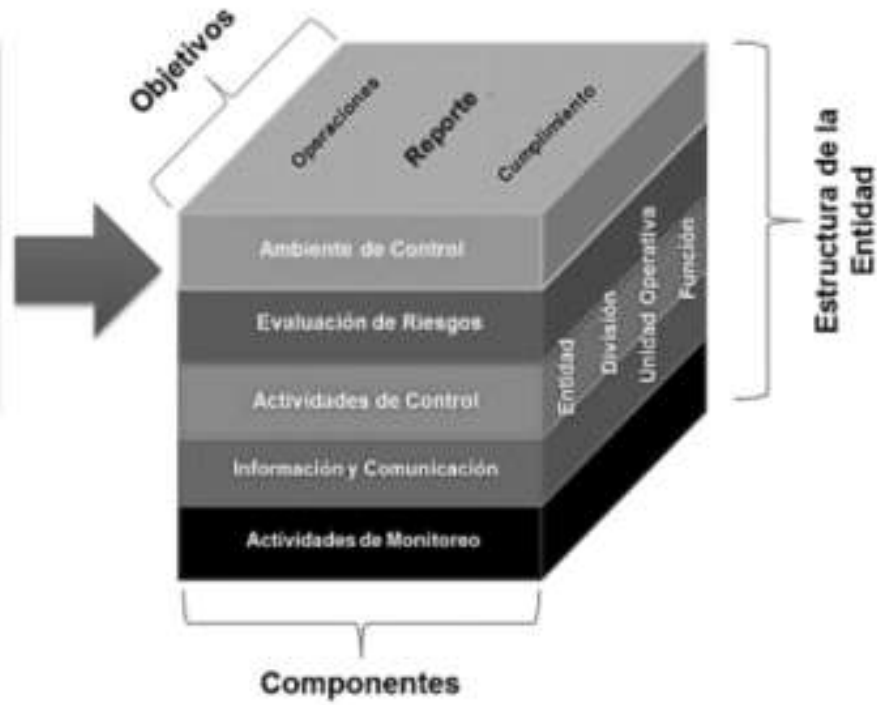

\section{Ambiente control}

El ambiente de control es el componente básico de la organización, es el cimiento en que se apoyan los demás componentes del control interno. Aporta disciplina y estructura y refleja la actitud general en la entidad, la conciencia y acciones de la administración y sus propietarios respecto a la importancia de los controles y el peso que ejercen en la determinación de las políticas, sus procesos y estructura organizacional.

El ambiente de control está integrado a su vez por los siguientes factores:

- Conciencia de control y estilo operativo. La administración es responsable de dirigir, controlar las operaciones, así como de establecer, comunicar, vigilar sus políticas y procedimientos. El ambiente de control está influido principalmente por las acciones y decisiones de la administración.

- La conciencia de control se refiere a la importancia que la administración le da al control interno. Es un concepto intangible que se puede definir como la actitud que la administración toma para asegurar que funcionen o no los controles.

En términos generales, la conciencia de control se refiere a las actitudes y acciones que la administración asume, con relación a:

- La importancia, cumplimiento y respecto de los controles

- Las debilidades o errores que se le informa 
- La atención que presta al sistema de información

- Las acciones que lleva a cabo ante o en condiciones inusuales

- La actitud que toma ante presiones de los accionistas para alcanzar determinados resultados.

Por lo tanto, para que la conciencia de control sea un aspecto eficaz del ambiente de control, la administración debe establecer controles apropiados y difundir la convicción sólida sobre la importancia de respetarlos. (Landázuri Cerón, 2016)

\section{Evaluación de riesgo}

Los riesgos son las acciones, eventos y circunstancias, internas o externas a la empresa que afectan su capacidad para lograr sus objetivos. Tomando en cuenta que los riesgos pueden afectar la existencia de la empresa, sin embargo, no existe una forma que nos garantice eliminarlos totalmente. Todas las entidades, independientemente de su tamaño, estructura, naturaleza o industria a la que pertenezcan, tienen riesgos en todos los niveles de su organización, la decisión de establecer una empresa es un riesgo.

La administración es la responsable de determinar el nivel de riesgo que su empresa puede aceptar y determinar acciones para mantenerlo en ese nivel. El nivel de riesgo apropiado para cada empresa varía en función de la naturaleza y circunstancia de cada negocio.

Las situaciones que generan riesgo en una empresa pueden ser:

- Cambios en el entorno de la entidad (nuevos reglamentos)

- Tecnología obsoleta

- Pérdida de mercado

- Dependencia hacía pocos clientes o proveedores

- Crecimiento o disminución acelerada

- Personal nuevo

- Ubicación geográfica de la empresa o sucursales.

- La naturaleza y complejidad de sus operaciones (materiales explosivos, químicos, etc.) (Landázuri Cerón, 2016)

\section{Actividades de control}

Este componente establece las políticas, disposiciones legales y procedimientos de control necesarios para gestionar y verificar la calidad de la gestión, su seguridad razonable con los requerimientos institucionales, para el cumplimiento de los objetivos y misión de los órganos, organismos, organizaciones y demás entidades. Se estructura en las siguientes normas.

- Coordinación entre áreas separación de tareas, responsabilidades y niveles de autorización.

- Documentación, registros oportunos y adecuados de las transacciones y hechos.

- Acceso restringido a los recursos activos y registros.

- Rotación del personal en las tareas claves.

- Control de las tecnologías de la información y las comunicaciones, indicadores de rendimiento y de desempeño. 
Existen dos tipos de controles:

Control preventivo. Son controles establecidos para evitar errores durante el desarrollo de las operaciones. Ejemplo la autorización de cheques a través de firmas mancomunadas.

Controles detectivos. Son controles para detectar y corregir los errores o las desviaciones que pueden ocurrir en el procesamiento de las operaciones. Funcionan sobre operaciones parcial o totalmente procesadas. Ejemplo, todo tipo de conciliaciones (bancarias, de cuentas por cobrar, etc.)

La efectividad de los controles establecidos en una entidad disminuye el grado de riesgo de posibles errores o irregularidades que afecten su información financiera.

Todas las empresas, sin importar el tamaño o la actividad que realicen, necesitan establecer controles que le ayuden a obtener una seguridad razonable de que se lograran los objetivos para los cuales fueron creadas. (Landázuri Cerón, 2016)

\section{Información y comunicación}

Es el proceso de capturar e intercambiar la información necesaria para conducir, administrar y controlar las operaciones de una entidad. La calidad tanto de la información como la comunicación de una entidad afectan la toma de decisiones oportunas, en el control de sus actividades y en la preparación de información financiera confiable.

\section{Información:}

Es el conjunto de datos generados por las operaciones y actividades (financieras y no financieras) que realiza una entidad. La información es necesaria en todos los niveles de la organización, para el logro de sus objetivos.

Existen varios sistemas de información, los cuales identifican, capturan, procesan los datos generados por las actividades y operaciones que realiza una entidad, también incluyen el reporte de los mismos, pueden ser computarizados, manuales o una combinación de los dos.

$\mathrm{Al}$ establecer los sistemas de información en una entidad, es necesario considerar, entre otros los siguientes aspectos:

- Deben aportar a la dirección los informes necesarios sobre el desempeño de la empresa.

- Los reportes generados por los sistemas de información deben emitirse con oportunidad y mantener suficientes detalles para que sean de utilidad al personal idóneo.

- Deben existir controles que aseguren y vigilen la participación de los usuarios en el desarrollo, actualización y prueba de los programas de cómputo.

- Debe existir controles que aseguren el acceso a la información sólo a personas autorizadas para ello. 


\section{Comunicación}

- La comunicación es el intercambio de información entre el personal idóneo para que descargue sus responsabilidades en tiempo y forma. La comunicación se realiza en todos los niveles de la organización a través de manuales de políticas, procedimientos de información financiera, memorandos, mensajes verbales y acciones de la administración.

- Un sistema efectivo de comunicación debe contener:

- Controles que aseguren la comunicación al personal de sus deberes y responsabilidades.

- Mecanismos y canales de comunicación para que el personal reporte sospechas sobre irregularidades.

- Controles para el manejo de situaciones inesperadas

- Controles para dar seguimiento oportuno a comunicaciones que recibe de compradores, proveedores, autoridades y otras entidades externas

- Controles que aseguren la comunicación de las normas éticas y política de la empresa tanto al personal como a entidades externas (compradores, proveedores). (Landázuri Cerón, 2016)

\section{Supervisión y monitoreo}

La supervisión es un proceso mediante el cual se comprueba, vigila y verifica el adecuado funcionamiento del sistema de control interno, a lo largo del tiempo, estas actividades de supervisión continua, se traducen en evaluaciones periódicas de los riesgos y de la eficacia de los procesos.

Las evaluaciones buscan identificar las debilidades de controles insuficientes para reforzarlos, eliminarlos e implantar nuevos.

Las deficiencias encontradas deben ser comunicadas a los niveles superiores y a los responsables directos de las mismas, para la aplicación de medidas correctivas.

Mediante el control interno se puede dar un grado de seguridad razonable no absoluta a la dirección, en cuanto a la fiabilidad de la información financiera y el cumplimiento de las leyes y normas aplicables y el consejo en cuanto al logro de los objetivos de la entidad, por lo que el éxito en su función se ve afectado por las limitaciones inherentes del control interno, que puede producir opiniones erróneas para la toma de decisiones.

El control interno puede deteriorarse por múltiples circunstancias y tiende con el tiempo a perder su efectividad, por lo que la administración es responsable de evaluar y verificar la validez y efectividad y ejercer sobre el mismo una supervisión permanente para producir los cambios que se requieran de acuerdo a las exigencias cambiantes del entorno económico y social en el que se desenvuelve la empresa.

Una supervisión permanente del sistema de control interno, determina la correcta interpretación y el cumplimiento de políticas, evalúa si los cambios realizados en las condiciones de operación, han causado procedimientos obsoletos o inadecuados y establece oportunamente si es necesario tomar medidas efectivas de corrección cuando se originen fallas en el mismo. (Landázuri Cerón, 2016) 


\section{Resultados.}

Esta investigación permite determinar mediante el análisis de fases propias de cada organización que integran el sector no financiero, la formulación de un cuestionario de control interno, que contiene el ambiente de control, componente, elemento y sub elemento, cargo directivo y nivel de la organización.

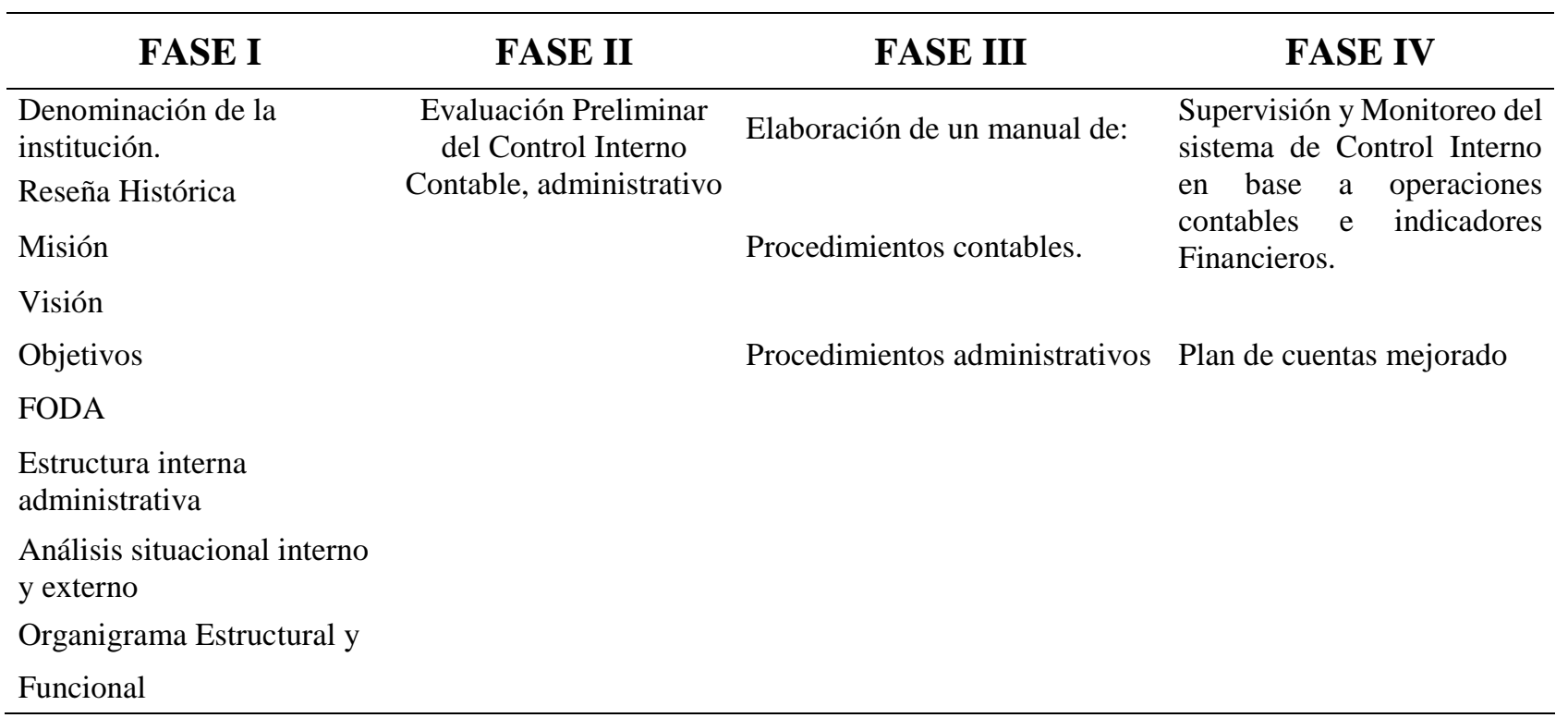

Fuente: Investigación Aplicada

Elaborado por: Los Autores

A partir de este diagnóstico y fundamentando en el análisis en cada área de la organización, se aplica el cuestionario de Control Interno que permite determinar las deficiencias y los ambientes a mejorar y monitorear. En el siguiente detalle de tablas se alinea los objetivos del grupo con los objetivos de las diferentes unidades de gobierno y ejecutoras de la Organizaciones del sector No financiero (ONF). Permite dar soporte a las actividades de planificación estratégica y Control Interno, con priorización de los objetivos permite fomentar que la gestión de riesgos pase a formar parte de la cultura del grupo.

Se propone el siguiente esquema en base a los estudios aplicados en la presente investigación, anclados a la normativa legal, políticas de control generadas por el ente de control, atribuciones y responsabilidades de cada órgano de gobierno estipuladas en la Ley Orgánica y su Reglamento. El esquema propuesto contiene: 48 preguntas para el Gerente, 43 al Contador, 49 al Presidente del Consejo de Administración, 17 al Presidente del Consejo de Vigilancia y 6 al Auditor Interno.

A continuación, se detalla por cada componente y cada área de relevancia en las ONF: 


\section{MODELO COSO I}

\section{AMBIENTE DE RIESGOS}

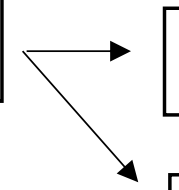

AMBIENTE INTERNO

,

ESTABLECIMIENTO DE

OBJETIVOS de Gerencia. El estatuto social y reglamento interno incluye los montos de autorización para adquisición, enajenación o gravamen de bienes inmuebles o la contratación de bienes o servicios. Conocer y resolver sobre los informes de Auditoría Interna y Externa. Decidir la distribución de los excedentes. Aprobar el reglamento que regule dietas, viáticos, movilización y gastos de representación del Presidente y directivos

CONSEJO DE ADMINISTRACIÓN: Planificar y evaluar el funcionamiento de la cooperativa. Aprobar políticas institucionales y metodologías de trabajo. Proponer a la asamblea reformas al estatuto social y reglamentos que sean de su competencia. Dictar los reglamentos de administración y organización internas. Aprobar Plan Estratégico, Plan Operativo y Presupuesto.

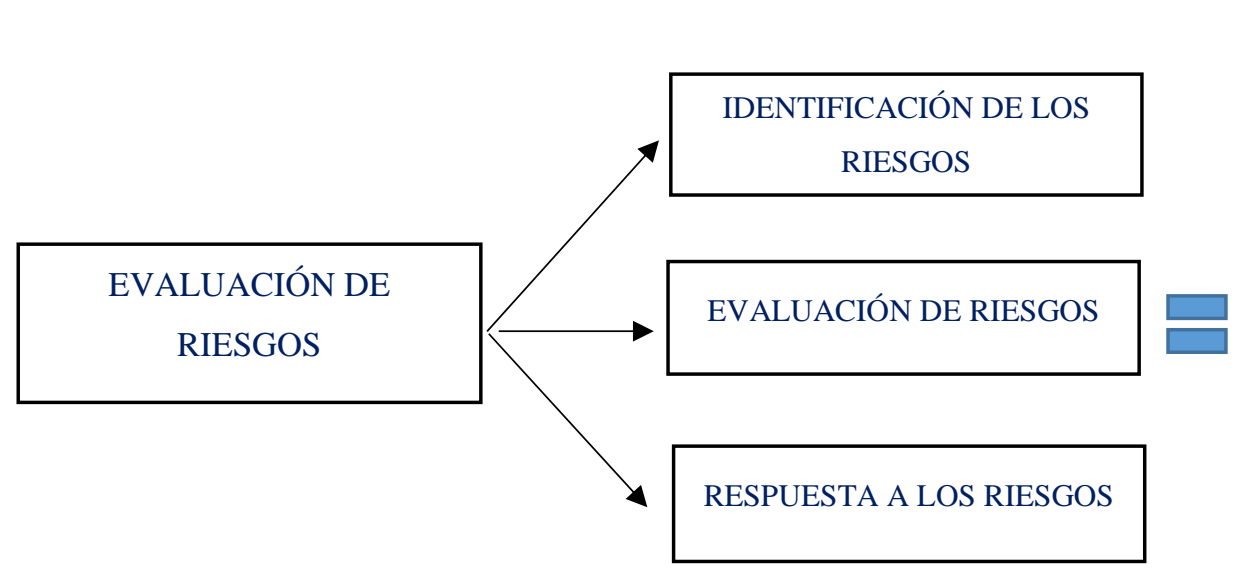

Fuente: Investigación Aplicada Elaborado por: Los Autores
CONSEJO DE VIGILANCIA: Para identificar los riesgos, el consejo de vigilancia efectúa las siguientes actividades: Controla las actividades económicas de la cooperativa. Vigila que la contabilidad de la cooperativa se ajuste a las normas técnicas y legales vigentes. Realizan controles concurrentes y posteriores sobre los procedimientos de contratación y ejecución. Vigila el cumplimiento de las recomendaciones formuladas por el auditor interno y externo. Informa al Consejo de Administración y a la Asamblea General sobre los riesgos que puedan afectar a la cooperativa.

Con el proceso formal para identificar los riesgos internos y externos de la organización. La administración realiza acciones alineadas a gestionar los riesgos importantes de la organización, como: Realizan arqueos sorpresivos y periódicos a los responsables de la custodia de efectivo y títulos valores, exigen al menos dos firmas en los cheques girados.

El proceso de valoración de riesgos de la entidad es un proceso para identificar y responder a los riesgos de la organización y los resultados que de ellos se derivan, para ello el Consejo de Vigilancia. Los riesgos importantes para la presentación de informes financieros incluyen eventos y circunstancias externas e internas que pueden ocurrir y afectar de manera adversa la habilidad de una entidad para iniciar, registrar, procesar e informar datos financieros. 

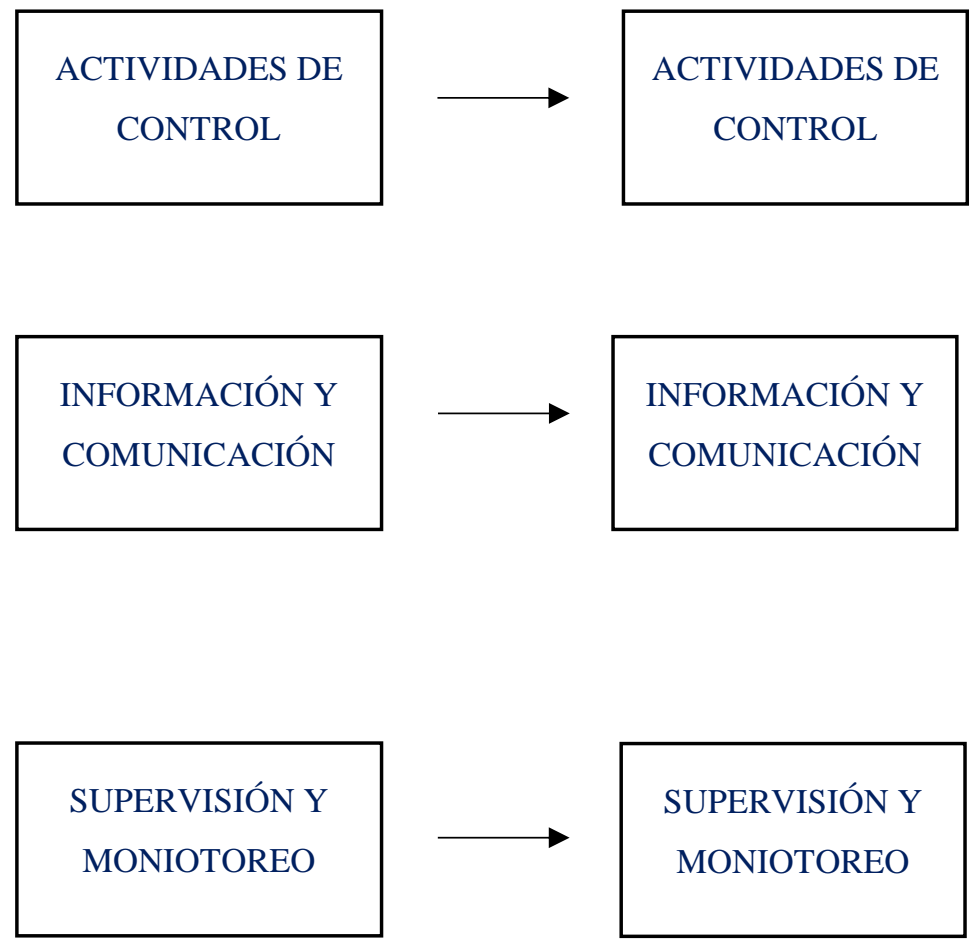

Fuente: Investigación Aplicada Elaborado por: Los Autores
GERENCIA: Como responsable de la marcha administrativa, operativa y financiera de la cooperativa, diseñar y administrar la política salarial de la cooperativa, en base a la disponibilidad financiera, adicional diseña y mantiene un sistema de control interno que asegure la gestión eficiente y económica de la cooperativa.

Presentación de los Informes de Gestión de los Consejos y Gerencia ante la Asamblea General. Presentación mensual de las gestiones efectuadas por Gerencia ante Consejo de Administración que permita determinar el \% de cumplimiento del POA y presupuesto. Actualización de la nómina de socios y certificados de aportación.

La estructura orgánica de la entidad, proporciona el marco dentro del cual se planifican ejecutan, controlan y supervisan las actividades para lograr los objetivos previstos. De acuerdo a la estructura organizacional, se encuentra claramente definidas las responsabilidades para cada nivel estructural de la organización, determinados en el manual de funciones. Estas políticas y procedimientos ayudan a asegurar que se cumplan con las directrices y normativa que regula la gestión de la organización. Para cada elemento y sub elemento se monitorea el cumplimiento (Asamblea General, Consejos y Gerencia)

Estos procedimientos valoran el diseño y la operación de los controles sobre una base oportuna y tomar las acciones correctivas necesarias, comunicando la información sobre fortalezas y debilidades que obtenidas en la aplicación del Control Interno. 


\section{CONCLUSIONES}

- Analizados y debatidos los datos obtenidos se ha llegado a las siguientes conclusiones:

- Se observa que, en las ONF, el impacto de una implementación de Control interno, es significativo por el grado de desactualización que tienen tanto en sus procedimientos como políticas contables, puesto que la gran mayoría de los encuestados carecen de conocimiento sobre los beneficios de un adecuado control, esto impedirá generar información útil, relevante, y razonable para la presentación oportuna de la información financiera.

- Es de ayuda la implementación de un sistema de control interno contable que les permita obtener información financiera fidedigna, razonable, y oportuna, para la preparación de estados financieros, evidenciándose que las operaciones contables no se llevan bajo parámetros específicos, que colaboren con la información financiera, y sea de ayuda para los usuarios de dicha información.

- La información financiera que se refleja de las operaciones contables no es razonable, razón por la cual es un factor preocupante, de modo tal que hay que reducir la incertidumbre y aumentar el conocimiento de quien se acerca a contemplar dicha información preparando información oportuna y fidedigna.

- El analizar los procesos de control interno, garantizan la utilización efectiva, económica, y eficiente de los recursos de la ONF, convirtiéndose en importante el tener un buen sistema de control interno debido a lo práctico que resulta al medir la eficiencia y la productividad al momento de implantarlos; en especial si se centra en las actividades básicas que las instituciones realizan, pues de ello dependen mejorar y generar crecimiento sostenido.

\section{BIBLIOGRAFIA}

- Blanco, Y. (2012). Auditoría Integral normas y procedimientos. Bogotà: Ecoe Ediciones.

- Cardozo, H. (s.f.). Auditoría del sector solidario: Aplicación de normas internacionales.Bogotà:Ecoe Ediciones.

- Casares, I. (27 de 03 de 2013).Informe coso I y coso II. Obtenido de https://www.auditool.

- Contralorìa General del Estado. (2003). Manual de Auditorìa de Gestión . Quito.CGE.

- Contraloría General del Estado. (2011). Guía Metodológica de la auditoría de gestión. Quito.CGE.

- De La Peña, A. (2009). Auditoría en enfoque práctico. Madrid : Paraninfo.

- Fonseca, O. (2007). Auditoría Gubernamental Moderna. Lima: IICO.

- Franklin, E. (2007). Auditoría Administrativa. México: Pearson.

- Ley Orgánica de la Economía Popular y Solidaria, R. O. (10 de mayo de

- 2011). Superintendencia de Economía Popular y Solidaria.

- Madariaga, J. (2004). Manual Práctico de Auditoría. Madrid: Deusto.

- Maldonado, M. (2011). Auditoría de Gestión. Quito. Abya-Yala.

- Mantilla, S. (2007). Control Interno Informe COSO . Bogotà: Ecoe Ediciones.

- Mantilla Blanco, S. A., \& Cante, S. Y. (2005). Auditoria del Control Interno. Bogota, Colombia: Ecoe Ediciones Ltda. 
- Normas Internacionales de Auditoría NIA 6. (2012). Evaluación del riesgo y control interno. Recuperado el 10 de Julio de 2012, de https://www.google.com.ec/?gws_rd=cr\&ei=S5dlUsLhNND_4APgq YGoDQ\#psj=1\&q=Normas+Internacionales+de+Auditoria+N\%C2\% $\mathrm{BA}+6+$ Evaluaci\%C3\%B3n+del+Riesgo+y+Control+Interno+

- Normas Internacionales de Información Financiera A-4. (1 de ENERO de 2006). Contaduria uady. Recuperado el 12 de Julio de 2013, de

- http://www.contaduria.uady.mx/files/material-

- Perdomo, A. (2004). Fundamentos de control interno (Novena edición ed.). México: Cengage.

- Santillana, J. R. (2003). Sistemas de control interno (Cuarta edicion ed.). Cengage Learning.

- Superintendencia de Economía Popular y Solidaria. (2012). Superintendencia de Economia Popular y Solidaria. Obtenido de http://www.seps.gob.ec/web/guest

- Zapata Sánchez, P. (2009). Contabilidad General (Quinta edición ed.). Colombia: Mc Graw Hill Interamericana S.A. 


\section{PARA CITAR EL ARTÍCULO INDEXADO.}

Chafla Granada, J., Solís Morejón, V. C., Sánchez Salazar, M., Carrillo Parra, E., \& Sánchez Chávez, R. (2019). La aplicación del control interno en las organizaciones de sector no financiero de la economía popular y solidaria del Ecuador. Ciencia Digital, 3(2.6), 464-480. https://doi.org/10.33262/cienciadigital.v3i2.6.592

\section{Ciencia \\ Digital \\ Editorial}

El artículo que se publica es de exclusiva responsabilidad de los autores y no necesariamente reflejan el pensamiento de la Revista Ciencia Digital.

El artículo queda en propiedad de la revista y, por tanto, su publicación parcial y/o total en otro medio tiene que ser autorizado por el director de la Revista Ciencia Digital.
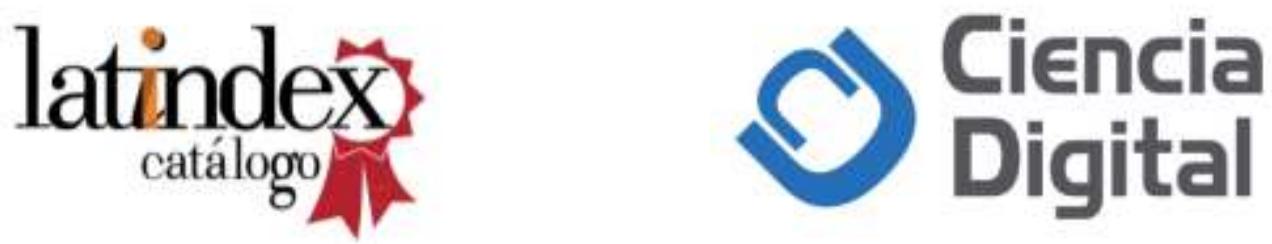